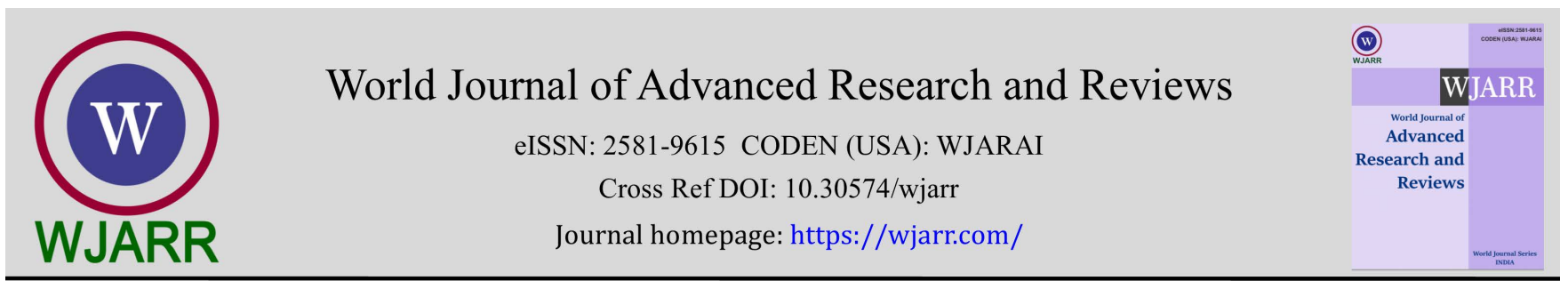

(RESEARCH ARTICLE)

Check for updates

\title{
Biological study of uro-genital trichomonosis in women during gynecological consultation at the prefectoral hospital of Macenta, Guinee
}

Boubacar Siddi Diallo 1, *, Boubacar Alpha Diallo 1, Ibrahima Conte 2, Moustapha Camara 3, Yaya Diallo 2, Abdourahamane Diallo ${ }^{2}$, Daniel Leno ${ }^{1}$, Ibrahima Sory Balde ${ }^{2}$, Telly Sy ${ }^{2}$, Yolande Hyjazi ${ }^{1}$ and Namory Keita ${ }^{1}$

${ }^{1}$ University Department of Gynecology Obstetrics, Donka national Hospital, Conakry Guinea.

${ }^{2}$ University Department of Gynecology Obstetrics, Ignace Deen national Hospital, Conakry Guinea.

${ }^{3}$ University Department of Pharmacy, Conakry Guinea.

World Journal of Advanced Research and Reviews, 2021, 10(03), 312-316

Publication history: Received on 17 May 2021; revised on 20 June 2021; accepted on 22 June 2021

Article DOI: https://doi.org/10.30574/wjarr.2021.10.3.0285

\begin{abstract}
Objectives: The objectives of this study were to: calculate the frequency of urogenital trichomoniasis, describe the socio-demographic characteristics and analyze the biological samples.

Methodology: This was a six (6) month cross-sectional prospective study carried out in the maternity ward (gynecological consultation) and in the laboratory of the Prefectural Hospital of Macenta. All the women who came for gynecological consultation were included in the study and among them, all those who presented a laboratory examination report for vaginal secretions and / or urine during the study period. Not included in the study were all the women who came for a gynecological consultation but who had not received a laboratory examination report for vaginal secretions and / or urine and all those already undergoing treatment to imidazole in the 15 days preceding the survey were not included. After applying the selection procedure (inclusion and non-inclusion criteria), we obtained a sample size $\mathrm{N}=703$ cases.
\end{abstract}

Results: The frequency of urogenital trichomoniasis was $26.46 \%$ among urogenital infections. The epidemiological profile was that of: young women aged 22-27 (42.47\%), housewife (39.76\%), having a primary education (30.43\%) and married (28.53\%). half of the patients were asymptomatic followed by those with fetid leucorrhoea $32.61 \%$. High positivity was found in vaginal secretions (30.41\%).

Conclusion: Urogenital trichomoniasis is a sexually transmitted infection, common in women of childbearing or childbearing age. It constitutes a public health problem, one of the control strategies for which it involves sensitization and care for women / couples at all levels of the health pyramid.

Keywords: Urogenital trichomonosis; Urogenital infection; Gynecological consultation

\section{Introduction}

Urogenital trichomoniasis is a sexually transmitted infection caused by a protozoan of the genus Trichomonas which is diagnosed more frequently in women than in men [1]. It is a cosmopolitan parasite that causes vaginitis manifested primarily by three symptoms: leucorrhoea, burns and vulvar pruritus [2]. Among the three species of Trichomonas parasites of man (Trichomonas vaginalis, Trichomonas tenax, Trichomonas intestinalis), only Trichomonas vaginalis is highly pathogenic, infesting the urogenital tract and possibly being responsible vaginitis and urethritis [3].

\footnotetext{
* Corresponding author: Boubacar Siddi Diallo

University Department of Gynecology Obstetrics , Donka national Hospital , Conakry Guinea.

Copyright (C) 2021 Author(s) retain the copyright of this article. This article is published under the terms of the Creative Commons Attribution Liscense 4.0.
} 
Transmission is sexual in the majority of cases. It affects both sexually active layers between 15 and 35 years old: $40 \%$ of women and $15 \%$ of men. About 25 to $85 \%$ of men are infected with Trichomonas vaginalis if their partner is. On the other hand, $100 \%$ of women are infected when their partners present with trichomoniasis [5]. Acute infections are characterized by severe vulvar pruritus, vaginitis, vulvitis, with dysuria and dyspareunia and bleeding spots on the mucosa (in $2 \%$ of patients) causing macular colitis or petechiae (raspberry-like cervix). In women, $50 \%$ of cases are asymptomatic [6]. Trichomonas parasitosis is ubiquitous; its incidence is estimated at 200 million cases per year worldwide [7].

WHO estimates that in Africa, the incidence of urogenital trichomoniasis now exceeds 180 million cases per year [8]. The frequency of the disease is particularly high in the African population (11 to $25 \%$ according to the surveys) as well as in the United States in the community of African origin up to $38 \%$ of the women who consult are carriers of Trichomonas vaginalis [9]. In Marseille (France), out of 346 women who came for consultation at the anti-venereal clinic, 165 were carriers of Trichomonas vaginalis [7]. In Senegal, more than 10\% of men and 12\% of women of childbearing age are victims [4; 8]. In Nigeria, according to OMEREE, 19\% of women are carriers of Trichomonas vaginalis [10]. In Guinea, the survey carried out by the AIDS project showed that out of 2,948 cervico-vaginal samples, $57.17 \%$ of STIs were found, including $8.86 \%$ of urogenital Trichomonas [1]. At the medico-communal center of Ratoma (Guinea) Sow B reported 33.28\% of urogenital trichomoniasis in 604 patients consulted [4].

The objectives of this study were to: calculate the frequency of urogenital trichomoniasis, describe the sociodemographic characteristics and analyze the biological samples.

\section{Methodology}

This was a six (6) month cross-sectional prospective study conducted in the maternity ward (gynecological consultation) and in the laboratory of the Prefectural Hospital of Macenta.

All the women who came for gynecological consultation were included in the study, and among them, all those who presented a laboratory examination report for vaginal secretions and / or urine during the study period.

All the women who came for a gynecological consultation but who had not received a laboratory examination report for vaginal secretions and / or urine and all those already undergoing treatment to imidazoles in the 15 days preceding the survey were not included.

After applying the selection procedure (inclusion and non-inclusion criteria), we obtained a sample of size $\mathrm{N}=703$ cases. The variables studied were:

- Epidemiological: frequency, age marital status, level of education, socio-professional category,

- $\quad$ Clinical and paraclinical: clinical signs and type of biological fluid

\section{Results}

\subsection{Frequency}

Out of 703 women recruited, we recorded 186 positive cases for trichomonas vaginalis, i.e., a frequency of $26.46 \%$.

Table 1 Frequency urogenital trichomonas

\begin{tabular}{|l|c|c|}
\hline \multicolumn{1}{|c|}{ Infection } & Number of cases & Percentage \\
\hline Urogenital trichomonas & 186 & 26.46 \\
\hline Gardnellosis & 273 & 38.83 \\
\hline Urogenital candidiasis & 179 & 25.46 \\
\hline Gonococcal & 65 & 9.25 \\
\hline Total & 703 & 100 \\
\hline
\end{tabular}




\subsection{Epidemiological characteristics}

\subsubsection{Age}

The 22-27 age group is the most concerned followed by that of $28-33$ years with respectively $42,47 \%$ and $29.46 \%$. The average age was 28 years with extremes of 10 years and 46 years old.

\subsubsection{Profession}

Women in consultation in our series are $39.76 \%$ of housewives.

\subsubsection{Level of education}

The majority of women in consultation had a primary level of education $30,43 \%$ followed by those out of school or $26.49 \%$.

\subsubsection{Marital status}

The study of this parameter reveals that $28.53 \%$ of women were married followed by single $28.28 \%$ and widows $16.98 \%$.

\subsection{Clinical and paraclinical variables}

\subsubsection{Clinical signs}

Half of the patients were asymptomatic followed by those with fetid leucorrhoea $32.61 \%$ and fetid pruritus or $25.23 \%$.

\subsubsection{Types of biological fluids}

The study of this parameter shows high positivity in vaginal secretions, ie $30.41 \%$ and $11.41 \%$ in urine.

Table 2 Types of biological fluids

\begin{tabular}{|l|c|c|c|}
\hline Pathological Products & $\begin{array}{c}\text { Number of Patients } \\
\text { sampled }\end{array}$ & $\begin{array}{c}\text { Number of positive } \\
\text { cases }\end{array}$ & Percentage \\
\hline Urine & 148 & 17 & 11.49 \\
\hline Vaginal secretions & 555 & 169 & 30.45 \\
\hline Total & 703 & 186 & 26.46 \\
\hline
\end{tabular}

\section{Discussion}

\subsection{Frequency}

Out of 703 women recruited, we recorded 186 positive cases for trichomonas vaginalis, ie a frequency of $26.46 \%$. This result is close to that reported by Sow B, i.e., 39.76\% [4].

\subsection{Epidemiological characteristics}

\subsubsection{Age}

The 22-27 age group is the most concerned followed by that of $28-33$ years with respectively $42 \%, 47 \%$ and $29.46 \%$. The average age was 28 years with extremes of 10 years and 46 years old. This higher rate in women of childbearing age could be justified by their intense sexual activity during this interval. The result recorded in young girls aged 10-15 could probably be due to the use of community showers or the same toilet effects as their parents.

\subsubsection{Profession}

Women in consultation in our series are $39.76 \%$ of housewives. 
This higher rate among housewives could be explained by their low level of knowledge of hygiene rules, unlike that of civil servants. Our result is lower than that found by Koné M.G which reports $58.48 \%$ of housewife in the maternity ward of the reference health center of the commune I Bamako-Mali [10].

\subsubsection{Level of education}

The majority of women in consultation had a level of primary education30, 43\% followed by those who did not attend school, i.e. $26.49 \%$. The analysis of this parameter shows that our result is superimposable on that found by R.BenAissa Ham Zanoui et al. or 37.60\% among women who had a primary education level [12].

\subsubsection{Marital status}

The study of this parameter reveals that $28.53 \%$ of women were married followed by single $28.28 \%$ and widows $16.98 \%$. Dieye. A.M et al. reported in their study 3.6\% among sex workers in Dakar [13].

\subsection{Clinical and paraclinical variables}

\subsubsection{Clinical signs}

Half of the patients were asymptomatic followed by those with fetid leucorrhoea $32.61 \%$ and fetid pruritus or $25.23 \%$.

\subsubsection{Types of biological fluids}

The study of this parameter shows high positivity in vaginal secretions, ie $30.41 \%$ and $11.41 \%$ in urine. This result is lower than that reported by G. Loua in his study at the Kindia regional hospital, ie $42.17 \%$ positivity in vaginal secretions [1].

\section{Conclusion}

Urogenital trichomoniasis is a sexually transmitted infection, common in women of childbearing or childbearing age. It constitutes a public health problem, one of the control strategies for it involves sensitization and care for women / couples at all levels of the health pyramid.

\section{Compliance with ethical standards}

\section{Disclosure of conflict of interest}

None.

\section{Statement of informed consent}

Informed consent was obtained from all individual participants included in the study.

\section{References}

[1] Loua G. Study of the frequency of urogenital trichomonas in women in gynecological consultation at the Kindia regional hospital. PhD thesis in pharmacy UGANC de Conakry. 2005; 50.

[2] Bernard P. Epidemiology of the sharp genital infections. SYNGOF 2005.Genital infections. Paris. P88.

[3] Porcheret H, L Maisonneuve, JL Jagot, V Esteve, Mple Pennec. Trichomonas tenax, review of respiratory diseases. February 2002; 19(1): 97-99.

[4] Gantier JC. Sexually transmitted diseases, Laboratory of biology and control of parasitized oranges. Chatenay Malabry Faculty of Pharmacy, Paris. 2005; 81.

[5] Petrin D, Delgaty K, Bhatt R, Garber G. Clinical and microbiological aspect of Trichomonas vaginalis. Clinical Microbiology Reviews. 1998; 11(2): 300-317.

[6] Gantier JC. Sexually transmitted diseases, Laboratory of biology and control of parasitized oranges". Chatenay Malabry Faculty of Pharmacy, Paris. 2005; 81. 
[7] World Health Organization. An overview of selected curable sexually transmitted dispase. Geneva, Swaziland. $1995 ; 2-27$

[8] Cravello L. Female genital infections. Leucorrhoea gynecology-obstetrics department B, conception hospital. page 55 Cedex Marseille. 2005.

[9] Omeree. Study of the frequency of pathological leucorrhoea in women during the period of genital activity at the outpatient unit in the obstetrics gynecology department at the Donka University Hospital. Doctoral thesis in Medicine UNGAN of Conakry. 1992; 21-25.

[10] Sow B. The frequency of urogenital trichomoniasis in women in gynecological consultation at the CMC of Matam. PhD thesis in pharmacy UGANC of Conakry. 2005; 38.

[11] Burch Ta, Rees Cw, Reardon Lv. Epidemiological studies on huntan trichomonas. The American journal of tropical medicine and hygiene. 1959; ${ }^{\circ} 8$ 312-318.

[12] R BenAissa-Hamzaoui, S Kouki, A Ben Hamida, A Kechrid, N Guedd. Assessment of the prevalence of sexually transmitted diseases in pregnant Tunisian women and validation of the clinical algorithm proposed by WHO for the care of patients.

[13] Dieye Am, Samb Nd, Ba A, Tardy M, Seck K, Wade As, Faye B. Evaluation of the efficacy of treatment of vaginal discharge according to the syndromic approach among sex workers in Dakar. 2005; 765. 